\title{
Automatic detection of sleep spindles using Teager energy and spectral edge frequency
}

\author{
Syed Anas Imtiaz, Siavash Saremi-Yarahmadi and Esther Rodriguez-Villegas
}

\begin{abstract}
Sleep spindles are the hallmark of N2 stage of sleep. They are transient waveforms observed on sleep electroencephalogram and their identification is required for sleep staging. Due to the large number of sleep spindles appearing on an overnight sleep EEG, automating the detection of sleep spindles would be desirable, not only to save specialist time but also for fully automated sleep staging systems. A simple algorithm for automatic sleep spindle detection is presented in this paper using only one channel of EEG input. This algorithm uses Teager energy and spectral edge frequency to mark sleep spindles and results in a sensitivity of $80 \%$ and specificity of about $98 \%$. It is also shown that more than $91 \%$ of spindles detected by the algorithm were in $\mathrm{N} 2$ and $\mathrm{N} 3$ stages combined.
\end{abstract}

\section{INTRODUCTION}

Human sleep is a dynamic process divided in to two distinct states that occur in alternate cycles: Nonrapid Eye Movement (NREM) and Rapid Eye Movement (REM). NREM stage is further divided into three stages, N1, N2 and N3 [1]. Sleep spindle is a micro-event of sleep electroencephalogram (EEG) which is characteristic of NREM stages of sleep. It is a transient waveform with waxing-waning morphology and exhibits strong presence in stage 2 of NREM sleep (N2), although it may be present in N3 stage with a lower frequency of occurence. According to the American Academy of Sleep Medicine, a sleep spindle is defined as "a train of distinct waves with frequency 11-16 $\mathrm{Hz}$ (most commonly 12-14 Hz) with a duration $\geq 0.5$ seconds" [1]. An example of typical sleep spindles in stage 2 of NREM sleep is shown in Fig. 1.

A typical night's sleep EEG recording contains 200-1000 spindles [2]. They are used by sleep specialists as one of the characteristic features when determining the appropriate stage of sleep. They need to be identified in order to mark the beginning and continuation of N2 phase of sleep. Scoring sleep spindles, however, is a laborious task and prone to human error. Indeed, a previous study has reported the inter-rater variability in scoring them to be around $80 \%$ [3].

Although the use of spindles for sleep staging is frequent and well established, its significance as a sleep event, otherwise, is less commonly known and is an area of active research. They are known to play a fundamental role in memory consolidation during sleep [4], as well as being related to the secretion of melatonin that helps in maintaining

S. A. Imtiaz, S. Saremi-Yarahmadi and E. Rodriguez-Villegas are with the Circuits and Systems Group, Electrical and Electronic Engineering Department, Imperial College London, United Kingdom. Email: (\{anas.imtiaz,siavash,e.rodriguez\}@imperial.ac.uk).

The research leading to these results has received funding from the European Research Council under the European Community's $7^{\text {th }}$ Framework Programme (FP7/2007-2013) / ERC grant agreement no. 239749. the body's circadian rhythms [5]. They are also understood to be sleep maintaining events having an active role in the progression of sleep to slow wave stages (N3). And it has also been suggested that they may be a relevant indicator for early stage development of CNS [6].

There have been various attempts to detect the presence of spindles in sleep EEG automatically as they are essential for any sleep staging system and their detection can also aid in research on their physiological role. Different research groups have tackled the problem of spindle identification with varying success. [7] and [8] used artificial neural networks and SVM classifiers with multiple features to achieve accuracy of about $89 \%$ and $95 \%$ respectively for each classifier. [9] used matching pursuit with multi-channel EEG and reported $81.6 \%$ sensitivity. [10] used bandpass filtering with thresholding, relative power and autoregressive modelling to achieve sensitivity and specificity values of $70.2 \%$ and $98.6 \%$. They also presented a standard assessment method to report performance of spindle detection (that will also be used in this paper). [11] used power thresholding and Hilbert-Huang transform to detect spindles in healthy children, using two EEG channels, and reported sensitivitiy and specificity of $88.2 \%$ and $89.7 \%$ respectively. [12] reported $96.2 \%$ sensitivity using maximum frequency in spindle range, Teager energy and harmonic decomposition of signals while [13] used amplitude-frequency normal modelling and reported $78.5 \%$ and $75.1 \%$ sensitivity for children and adult spindle detection respectively.

In this paper, a simple and low complexity method is presented for the automatic detection of sleep spindles, using only a single channel EEG data, that is suitable to be used as part of a sleep staging system or a spindle detector on its own. A brief overview of the methods and data used is given in Section II along with the description of each step in the spindle detection algorithm. The results are given in Section III and further discussed in Section IV including a comparison of results with other algorithms that have been tested on the same database.

\section{MAterial \& Methods}

\section{A. Database}

Polysomnography data from the DREAMS Sleep Spindles Database of University of MONS - TCTS Laboratory and Universite Libre de Bruxelles - CHU de Charleroi Sleep Laboratory [14] was used for testing the algorithm. The data consists of 30-minute excerpts from six subjects (3 males and 3 females, average age 45.7 years) with various sleep pathologies. The excerpts have been marked visually by two 


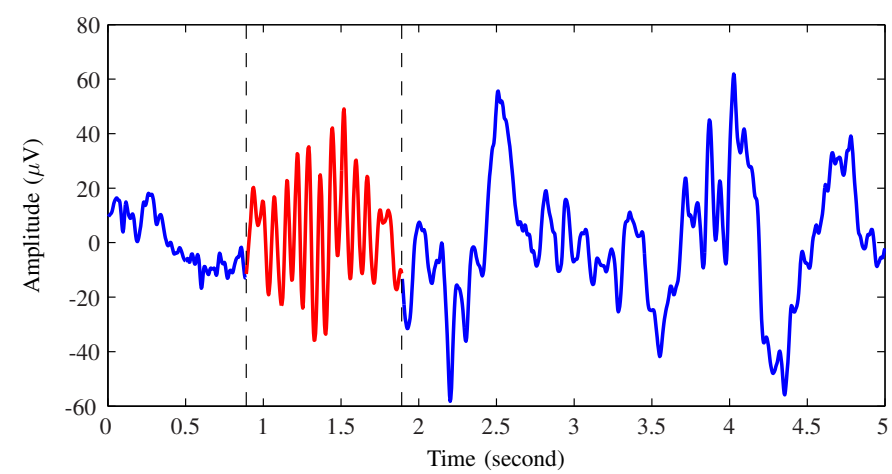

Fig. 1: A typical sleep spindle (between dashed lines)

scorers for sleep spindles. Data was originally recorded using two EOG, three EEG and one EMG channels. The sampling frequency is $200 \mathrm{~Hz}$ and the annotated channel is CZ-A1 except for subjects 1 and 3, for whom the annotated channel is $\mathrm{C} 3-\mathrm{A} 1$ and the sampling frequency $100 \mathrm{~Hz}$ and $50 \mathrm{~Hz}$ respectively. All signals were first resampled to have a uniform sampling frequency of $256 \mathrm{~Hz}$ using the MATLAB function 'resample'. Of the entire 180 minutes of data available for testing, an average of 56\% was part of NREM2 stage sleep, confirmed by the hypnogram provided with the data. No attempt was made to exclude either noisy data segments, or data from any other stages of sleep or wakefulness.

\section{B. Methods}

1) Teager Energy Operator: Teager energy operator (TEO) is a non-linear operator that can estimate the energy of a signal on-the-fly [15], [16]. It is particlarly useful in highlighting the abrupt transitions in a signal while suppressing the soft transitions. For a discrete-time signal, its Teager energy is computed using the following equation [16]:

$$
\psi[x(n)]=x^{2}(n)-x(n+1) x(n-1)
$$

Teager energy operator, $\psi$, when applied to polysomnographic signals, appropriately filtered for sleep spindle detection, demonstrates a rise in energy level when a spindle appears. The sudden change in frequency and the waxing and waning amplitude of sleep spindles is well tracked by the Teager energy operator.

2) Spectral Edge Frequency - 50\%: Spectral edge frequency at 50\% (SEF50) is the frequency below which half of the signal power is present. This is equivalent to the median frequency of the signal. It can be computed from the magnitude of FFT coefficients ( $\mathrm{mag}$ ) using (2), where $n$ is the total number of FFT coefficients and $x$ is the index to solve the equation for. The required frequency is then the $x_{t h}$ frequency from the array of FFT frequency components. In this work, SEF50 was analyzed in the $8-15 \mathrm{~Hz}$ frequency range since it covers both alpha $(8-13 \mathrm{~Hz})$ and spindle frequency range. It was found that spindle-like alpha rhythms have lower median frequency in this range and therefore this feature was used to reduce the number of false detections.

$$
\begin{array}{r}
\sum_{i=1}^{x}|m a g|^{2}=0.50 \times \sum_{i=1}^{n}|m a g|^{2} \\
S E F 50=\operatorname{freq}(x)
\end{array}
$$

\section{Spindle Detection Algorithm}

A block diagram of the complete spindle detection algorithm is shown in Fig. 2. EEG input signal is first filtered using a first order high-pass filter with a cut-off frequency of $0.16 \mathrm{~Hz}$, followed by a second order low-pass filter with $50 \mathrm{~Hz}$ as the cut-off frequency at the preprocessing stage. This bandlimited signal is used as input to the main spindle detection algorithm. The input signal is then filtered with a fourth order Butterworth band-pass filter with lower and upper cutoff frequencies $11 \mathrm{~Hz}$ and $16 \mathrm{~Hz}$ respectively, which is the spindle frequency range. This step gets rid of all the frequency content that is not of interest for spindle detection. This filtered signal is then segmented in to epochs of 0.25 seconds with $50 \%$ overlap between successive ones. Teager Energy of the filtered signal is then determined in each epoch using (1). If the Teager energy values of all samples within the epoch are greater than a certain varying threshold, the epoch is marked as a candidate spindle. Because of the overlapping epochs a potential sleep spindle in one epoch may have started in the previous epoch or carried over in to the next epoch. For this reason, when an epoch is marked as a candidate spindle, the epochs immediately preceeding and succeeding the current epoch are also marked as part of the current spindle thus creating a candidate spindle zone.

The threshold for an epoch to be marked as a valid candidate spindle was determined by taking the mean value of the Teager energy over 60 previous epochs. The threshold was then established as 2.19 times this mean value i.e. all samples in an epoch had to be greater than the running mean of last 60 epochs by a factor of 2.19 to be considered as a valid spindle. The number of epochs and multiplication factor for threshold were determined empirically. Establishing the threshold based on the data itself obviates the need for any manual patient specific adjustments.

At this stage, minimum and maximum spindle duration constraints were also applied prior to spectral analysis. If the duration of a candidate spindle was found to be greater than 3 seconds or less than 0.5 seconds, the candidate was discarded and not subject to any further analysis.

The next stage in the algorithm is enabled only when a candidate spindle is detected at first stage and obeys the duration constraints. Frequency content of each epoch in the preprocessed signal, corresponding to the epoch in candidate spindle zone, is analysed using a 512-point Fast Fourier Transform (FFT). Spectral edge frequency (SEF50) for each epoch in the $8-15 \mathrm{~Hz}$ band is computed using (2) and its average determined for all epochs in the candidate zone. If SEF50 was found less than a fixed threshold the candidate spindle 


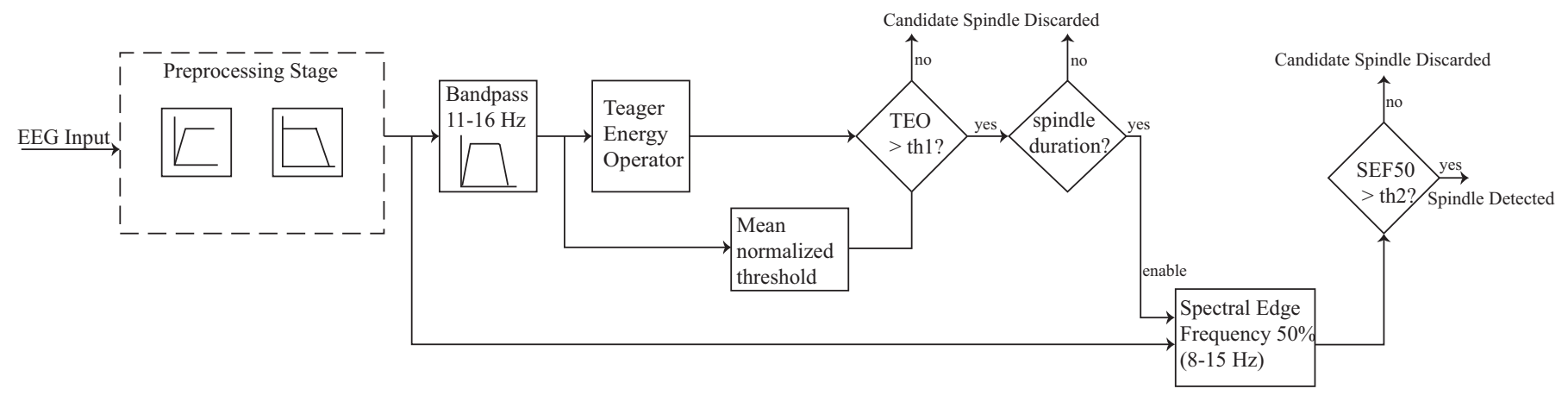

Fig. 2: Block diagram of sleep spindle detection algorithm

was rejected otherwise the candidate spindle was deemed to be a positive detection. This stage is highly specific and helps in removing false spindles and alpha rhythms that may have been erroneously detected at the first stage. The threshold for spectral edge frequency (SEF50) was fixed at $10.7 \mathrm{~Hz}$ for all test cases. This was determined experimentally by analysing the frequency content of true spindles and false detections from the first stage.

The output from each stage of the algorithm is shown in Fig. 3 where the Teager energy block identifies four candidate spindles. One of them is a false detection which is subsequently rejected by analysing the frequency of the candidate spindles.

\section{RESULTS}

The spindle detection algorithm proposed in this paper was tested on 30-minute EEG excerpt from six subjects. The union of spindles scored visually by two scorers was taken as the reference to compare the results against. An automatically detected spindle is marked as True Positive (TP) if it overlaps at least partially with the reference spindle at that time. If no point of the detected spindle overlaps with the reference, it is considered as a false detection and marked as False Positive (FP), while the number of spindles that went undetected by the algorithm were classified as False Negatives (FN). The number of True Negatives (TN) was approximated using the method proposed in [10] with the following equation:

$$
T N=\frac{\text { Total record duration }}{\text { Avg. detected spindle duration }}-T P-F P-F N
$$

The average duration of the detected spindles was computed separately for each subject to determine the True Negatives.

The performance of this algorithm was characterized by finding the Sensitivity and Specificity using (4) and (5) for each subject individually as well as for all of them combined. The results are shown in Table I with the total number of spindles for each subject and the fraction of those correctly identified by the algorithm.

$$
\text { Sensitivity }=\frac{T P}{T P+F N}
$$

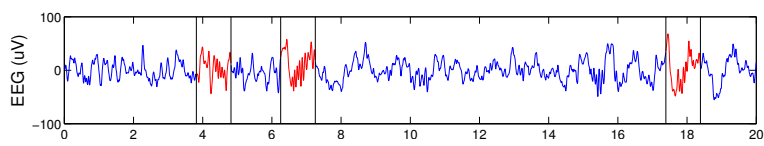

(a)

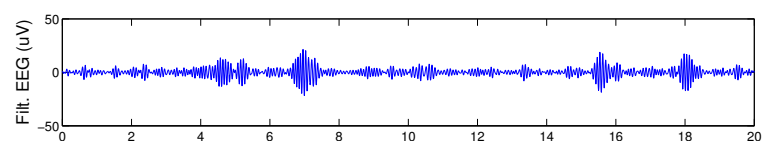

(b)

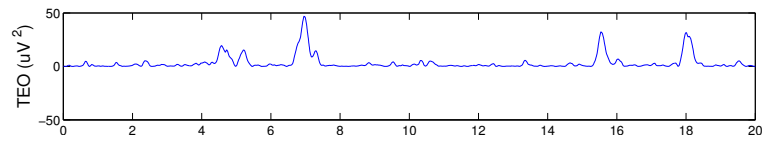

(c)

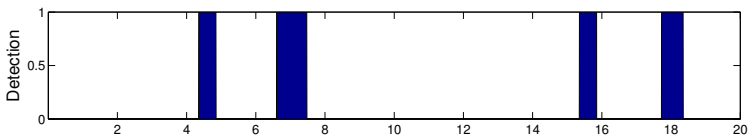

(d)

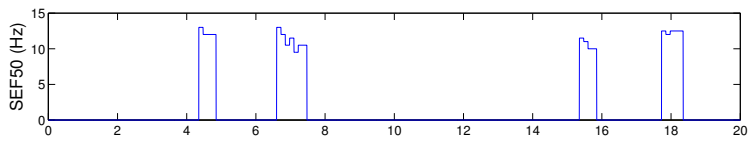

(e)

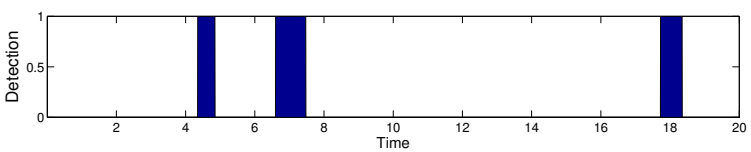

(f)

Fig. 3: (a) EEG input with three spindles marked between vertical lines; (b) 11-16 Hz filtering output; (c) TEO output; (d) candidate spindles; (e) SEF50 for each epoch in the candidate spindle zone; (f) correctly detected spindles

$$
\text { Specificity }=\frac{T N}{T N+F P}
$$

\section{DISCUSSION}

The algorithm was tested on sleep data excerpts that included all sleep stages to reflect real world conditions. Of the 
TABLE I: Spindle detection algorithm performance

\begin{tabular}{ccccc}
\hline Subject & Total Spindles & True Pos. & Sens. (\%) & Spec. (\%) \\
\hline 1 & 134 & 111 & 82.8 & 96.7 \\
2 & 77 & 58 & 75.3 & 98.3 \\
3 & 44 & 39 & 88.6 & 97.7 \\
4 & 63 & 38 & 60.3 & 97.8 \\
5 & 103 & 87 & 84.5 & 97.1 \\
6 & 117 & 99 & 84.6 & 98.1 \\
\hline All & 538 & 432 & 80.3 & 97.6 \\
\hline
\end{tabular}

538 visually scored sleep spindles, the algorithm successfully detected 432 spindles yielding a sensitivity of $80.3 \%$ within $\pm 3.36 \%$ for a $95 \%$ confidence interval. The rejection of most of the background EEG as true negatives also leads to a high specificity of almost $98 \%$.

The results in Table I show that the algorithm performance is similar for all test cases except for a lower sensitivity in the case of subject 4 . Further analysis shows that this subject has a high Wake stage content (30\%) in the recording. Additionally, almost half of the spindles visually scored in this record were found to be part of the Wake stage when compared against the hypnogram provided with the database. The algorithm falsely detected only 14 of the 31 spindles in Wake stage while the other 24 true positive detections were part of NREM stages. With the Wake stage removed from analysis, sensitivity for subject 4 goes up to $75 \%$.

The spindles detected were further analyzed to determine the corresponding sleep stage. Table II shows the total number of sleep spindles detected by the algorithm for each subject and the number of detections in each sleep stage (classified according to AASM [1] rules). It can be seen that most of the sleep spindles detected were from N2 stage (about 69\%) and more than $91 \%$ of spindles detected were from stages N2 and N3 combined. These are also the two stages where sleep spindles are most often observed. We believe that together with using the metrics proposed in [10] it is important to report the fraction of spindles detected in each stage of sleep as well to evaluate the performance of any spindle detection algorithm.

TABLE II: Sleep Spindles (SS) detected in each sleep stage

\begin{tabular}{rrrrrrr}
\hline Sub & $\mathrm{SS}_{\text {tot }}$ & $\mathrm{SS}_{W}$ & $\mathrm{SS}_{N 1}$ & $\mathrm{SS}_{N 2}$ & $\mathrm{SS}_{N 3}$ & $\mathrm{SS}_{R}$ \\
\hline 1 & 190 & 10 & 3 & 135 & 42 & 0 \\
2 & 101 & 0 & 0 & 77 & 24 & 0 \\
3 & 91 & 5 & 8 & 75 & 3 & 0 \\
4 & 104 & 32 & 8 & 54 & 10 & 0 \\
5 & 164 & 4 & 0 & 110 & 50 & 0 \\
6 & 146 & 0 & 0 & 97 & 49 & 0 \\
Total & 796 & 51 & 19 & 548 & 178 & 0 \\
\hline
\end{tabular}

Comparison of results against [10] and [13] (evaluated using the same dataset) shows superior sensitivity and similar specificty values. These are shown in Table III.

\section{CONCLUSiON}

Identification of sleep spindles is an integral part of sleep staging. Automatic detection of sleep spindles is desirable to aid development of automatic sleep staging systems and
TABLE III: Comparison of this work with other algorithms

\begin{tabular}{rrr}
\hline Method & Sens. (\%) & Spec. $(\%)$ \\
\hline$[10]$ & 70.2 & 98.6 \\
{$[13]$} & 75.1 & 96.7 \\
This work & 80.3 & 97.6 \\
\hline
\end{tabular}

reduce the manual workload of sleep technicians. In this paper a simple automatic sleep spindle detection algorithm is presented that makes use of the Teager energy operator to isolate candidate spindle zones on sleep EEG followed by spectral edge frequency in $8-15 \mathrm{~Hz}$ band to confirm their presence. The algorithm uses a normalized threshold and, therefore, requires no patient-specific adjustments. It demonstrates good performance with sensitivity and specificity values of $80 \%$ and $97.6 \%$ respectively.

\section{REFERENCES}

[1] C. Iber, S. Ancoli-Israel, A. Chesson, and S. Quan, Eds., The AASM manual for the scoring of sleep and associated events: rules, terminology and technical specifications. Westchester, IL: American Academy of Sleep Medicine, 2007.

[2] E. Huupponen, G. Gómez-Herrero, A. Saastamoinen, J. Vrri, A. Hasan, and S. Himanen, "Development and comparison of four sleep spindle detection methods," Artif. Intell. Med., vol. 40, no. 3, pp. 157-170, 2007.

[3] K. Campbell, A. Kumar, and W. Hofman, "Human and automatic validation of a phase-locked loop spindle detection system," Electroencephalogr. and Clin. Neurophysiol., vol. 48, no. 5, pp. 602-5, 1980.

[4] S. M. Fogel and C. T. Smith, "The function of the sleep spindle: A physiological index of intelligence and a mechanism for sleep-dependent memory consolidation," Neurosci. Biobehav. R., vol. 35, no. 5, pp. 1154 $1165,2011$.

[5] L. D. Gennaro and M. Ferrara, "Sleep spindles: an overview," Sleep Med. Rev., vol. 7, no. 5, pp. 423-440, 2003.

[6] M. Schabus, K. Hödlmoser, G. Gruber, C. Sauter, P. Anderer, G. Klsch, S. Parapatics, B. Saletu, W. Klimesch, and J. Zeitlhofer, "Sleep spindlerelated activity in the human eeg and its relation to general cognitive and learning abilities," Eur. J. Neurosci., vol. 23, no. 7, pp. 1738-1746, 2006.

[7] D. Gorur, U. Halici, H. Aydin, G. Ongun, F. Ozgen, and K. Leblebicioglu, "Sleep spindles detection using short time fourier transform and neural networks," in IEEE IJCNN, Honolulu, May 2002.

[8] N. Acır and C. Güzeliş, "Automatic recognition of sleep spindles in eeg by using artificial neural networks," Expert Sys. Appl., vol. 27, no. 3, pp. 451-458, 2004.

[9] S. V. Schönwald, E. L. de Santa-Helena, R. Rossatto, M. L. Chaves, and G. J. Gerhardt, "Benchmarking matching pursuit to find sleep spindles," J. Neurosci. Methods, vol. 156, no. 1-2, pp. 314-321, 2006.

[10] S. Devuyst, T. Dutoit, P. Stenuit, and M. Kerkhofs, "Automatic sleep spindles detection overview and development of a standard proposal assessment method," in IEEE EMBC, Boston, September 2011.

[11] L. Causa, C. M. Held, J. Causa, P. A. Estévez, C. A. Perez, R. Chamorro, M. Garrido, C. Algarín, and P. Peirano, "Automated sleep-spindle detection in healthy children polysomnograms," IEEE Trans. Biomed. Eng., vol. 57, no. 9, pp. 2135-46, 2010.

[12] F. Duman, A. Erdamar, O. Erogul, Z. Telatar, and S. Yetkin, "Efficient sleep spindle detection algorithm with decision tree," Expert Sys. Appl., vol. 36, no. 6, pp. 9980-9985, 2009.

[13] A. Nonclercq, C. Urbain, D. Verheulpen, C. Decaestecker, P. Van Bogaert, and P. Peigneux, "Sleep spindle detection through amplitudefrequency normal modelling," J. Neurosci. Methods, vol. 214, no. 2, pp. 192-203, 2013.

[14] University of MONS - TCTS Laboratory. (2013) The DREAMS Sleep Spindles Database. [Online]. Available: http://www.tcts.fpms.ac. be/ devuyst/Databases/DatabaseSpindles/.

[15] J. Kaiser, "On a simple algorithm to calculate the 'energy' of a signal," in IEEE ICASSP, Albuquerque, April 1990.

[16] J. Kaiser, "Some useful properties of teager's energy operators," in IEEE ICASSP, Minneapolis, April 1993. 